\title{
Conservación
}

\section{Aves del Parque Nacional Los Mármoles, Hidalgo, México}

\author{
Birds of Los Mármoles National Park, Hidalgo, Mexico
Omar Larios-Lozano, Jorge Valencia-Herverth, Jessica Bravo-Cadena, Erika Guzmán-Arias y Raúl Ortiz-Pulido*

Centro de Investigaciones Biológicas, Instituto de Ciencias Básicas e Ingeniería, Universidad Autónoma del Estado de Hidalgo, Km 4.5 carretera Pachuca-Tulancingo, 42184 Mineral de la Reforma, Hidalgo, México

Recibido el 3 de agosto de 2016; aceptado el 20 de junio de 2017

Disponible en Internet el 6 de diciembre de 2017

\begin{abstract}
Resumen
Conocer la diversidad y distribución de las especies de aves de un área natural protegida contribuye a desarrollar acciones de conservación. En este trabajo se presenta el listado de aves del Parque Nacional Los Mármoles, Hidalgo, México. Para obtenerlo se conjuntaron observaciones de 5 años de muestreo. Para el registro de las especies se emplearon puntos de conteo y trayectos para las aves diurnas, y estaciones de escucha para las aves con actividad nocturna, registrándose un total de 195 especies de aves. Del total de especies 137 fueron catalogadas como residentes permanentes, 44 como migratorias invernales, 6 como migratorias de verano y 8 como transitorias. El mayor número de especies se registró en el Bosque de Pinus-Quercus (91 especies), seguido por el Bosque de Juniperus (64) y el bosque de Quercus (57). Se registraron 15 especies bajo alguna categoría de riesgo y 26 especies con algún grado de endemismo para México. La diversidad registrada del parque equivale al $38 \%$ de la avifauna del estado de Hidalgo y cerca del $17 \%$ de la avifauna de México.
\end{abstract}

(C) 2017 Universidad Nacional Autónoma de México, Instituto de Biología. Este es un artículo Open Access bajo la licencia CC BY-NC-ND (http://creativecommons.org/licenses/by-nc-nd/4.0/).

Palabras clave: Listado de aves; Inventario avifaunístico; Parque Nacional

\section{Abstract}

Knowing the diversity and distribution of birds from a natural protected area can contribute to the development of conservation actions. Here we report the check-list of bird observed in Los Mármoles National Park, Hidalgo, Mexico. Count points and transects were used for recording diurnal birds, and listening stations for nocturnal birds; 195 species were registered. The total species richness included 137 resident species, 44 winter visitors, eight transient migrants, and six summer migrants. Richness varied between vegetation (forest) types, with 91 species found in Pinus-Quercus forests, 64 species in Juniperus forests, and 57 species in Quercus forests. Fifteen species are under some risk category and 26 species were endemic to Mexico. The diversity registered in Los Mármoles represents $38 \%$ of the birds known from the Hidalgo State and nearly $17 \%$ of the birds known from Mexico.

(C) 2017 Universidad Nacional Autónoma de México, Instituto de Biología. This is an open access article under the CC BY-NC-ND license (http://creativecommons.org/licenses/by-nc-nd/4.0/).

Keywords: Bird check-list; Bird inventories; National Park

\footnotetext{
* Autor para correspondencia.

Correo electrónico: raulortizpulido@yahoo.com (R. Ortiz-Pulido).

La revisión por pares es responsabilidad de la Universidad Nacional Autónoma de México.
} 


\section{Introducción}

Las áreas naturales protegidas (ANP) han sido un elemento primordial para la conservación a nivel mundial, pues su función principal ha sido minimizar los riesgos de extinción de muchas especies y proporcionar protección a la diversidad biológica que se encuentre en ellas (Margules y Sarkar, 2009). En México, la implementación del Sistema Nacional de ANP ha tenido un impacto positivo en la conservación de la diversidad biológica y los servicios ambientales que proporcionan a la sociedad (Ceballos y Márquez, 2000).

México cuenta con 177 ANP federales que cubren el 7.8\% de la superficie terrestre e insular del país, 66 de las cuales son Parques Nacionales (Conanp, 2015). La mayoría de los Parques Nacionales en México fueron decretados antes de la década de 1970 principalmente para la protección de los recursos forestales y paisajísticos. Sin embargo, hasta la última década del siglo xx no hubo avances significativos en el conocimiento de la biodiversidad que resguardan (Bezaury-Creel et al., 2009).

En el estado de Hidalgo se han decretado 5 ANP de carácter federal: Parque Nacional El Chico, Parque Nacional Los Mármoles (PNM), Parque Nacional Tula, Área de Protección de Recursos Naturales Zona Protectora Forestal Vedada Cuenca Hidrográfica del Río Necaxa y la Reserva de la Biosfera Barranca de Metztitlán (RBBM). En conjunto, estas ANP representan el $6.29 \%$ de la superficie del estado (Conanp, 2015). Además, se encuentra en proyecto de decreto el Corredor Biológico Bosque Mesófilo de Montaña, el cual aumentaría la superficie bajo protección federal (Conanp, 2015). Desafortunadamente, estas ANP cuentan con pocos estudios actualizados sobre los vertebrados que albergan (e.g., Cruz-Elizalde, Ramírez-Bautista, Wilson y Hernández-Salinas, 2015; Hernández-Flores y Rojas-Martínez, 2010), entre ellos las aves (e.g., Ortiz-Pulido et al., 2010; Valencia-Herverth, Ortiz-Pulido y Enríquez, 2012; Valencia-Herverth et al., 2012b).

Las aves del estado de Hidalgo han sido estudiadas de manera formal desde mediados del siglo XIX (Villada, 1865). Sin embargo, los avances en su conocimiento han sido paulatinos durante el siglo xx (Rodríguez-Yáñez, Villalón y Navarro, 1994). En cambio, en lo que va del siglo XXI se ha desarrollado un mayor interés por conocer de mejor manera la avifauna de diversas regiones del estado. Ejemplo de ello son los trabajos de Martínez-Morales (2001, 2004) y MartínezGarcía, (2006), que, entre otros estudios, ayudaron a elaborar la primer compilación de información sobre el estatus de las aves en esta entidad (Martínez-Morales et al., 2007). Posteriormente, se han realizado avances en distintas zonas de Hidalgo y áreas del conocimiento ornitológico (Carbó-Ramírez y Zuria, 2011; Martínez-Morales, Mendiola, Zuria, Chávez y Campuzano, 2013; Ortiz-Pulido et al., 2010).

En las ANP del estado de Hidalgo hasta hace pocos años solo se contaba con inventarios parciales (Gallina, González, Mortal y Tello, 1974; Mancilla, 1988; SánchezMejorada, 1978). Sin embargo, en la última década se ha incrementado este conocimiento principalmente en la RBBM
(Ortiz-Pulido et al., 2010; Valencia-Herverth et al., 2012a; Valencia-Herverth et al., 2012b) y el Parque Nacional el Chico (García y Ortiz-Pulido, 2007; Ortiz-Pulido, MauricioLópez, Martínez-García y Bravo-Cadena, 2008). Sin embargo, aún se carece de datos precisos para las otras 3 ANP del estado.

El PNM es una de las ANP más antiguas en el estado de Hidalgo, ya que fue decretada desde 1936. Para esta área solo se tienen registros esporádicos de algunas especies de aves (Aquila chrysaetos, Patagioenas fasciata y Myiarchus nuttingi), en estudios que tiene más de 50 años (Amadon y Phillips, 1947; Friedmann, Griscom y Moore, 1950; Leopold, 1965; Mengel y Warner, 1948; Sutton y Burleigh, 1940). Existen 2 estudios con aves que han sido desarrollados cerca del PNM. El primero de ellos fue realizado a finales de la década de 1930 por Sutton y Burleigh (1941), quienes siguiendo la carretera Panamericana registraron 126 especies de aves en los municipios de Chapulhuacán, Tasquillo y Zimapán. El segundo estudio fue llevado a cabo en varias localidades del municipio de Jacala de Ledezma, donde se registraron 57 especies en diferentes tipos de vegetación (Valencia-Herverth y Valencia-Herverth, 2008).

Considerando lo anterior, el objetivo general de este estudio fue conocer las especies de aves que habitan el PNM, buscando aportar información básica sobre su presencia, abundancia, distribución, estacionalidad y situación de riesgo. Creemos que esto aportará información sólida que podrá ser usada por los tomadores de decisiones y por quienes elaboren acciones de conservación, educación ambiental y manejo en el PNM y zonas cercanas.

\section{Materiales y métodos}

El PNM se encuentra en la parte noroeste del estado de Hidalgo, en la región conocida como sierra Gorda $\left(20^{\circ} 45^{\prime} 39^{\prime \prime}\right.$ $20^{\circ} 58^{\prime} 22^{\prime \prime} \mathrm{N}, 99^{\circ} 08^{\prime} 57^{\prime}$ - $99^{\circ} 18^{\prime} 39^{\prime}$ O, fig. 1) y su territorio incluye los municipios de Jacala de Ledezma, Nicolás Flores, Pacula y Zimapán (Conanp, 2007). Cuenta con una superficie de 23,150 ha y tiene un intervalo altitudinal que va de los 870 a los $3,000 \mathrm{~m} \mathrm{snm}$. Se localiza en la provincia fisiográfica denominada Sierra Madre Oriental y en la subprovincia Carso Huasteco, que se caracteriza por presentar rocas sedimentarias del Cretácico, del Jurásico y metamórficas del Paleozoico (Espinoza, Aguilar y Ocegueda, 2004).

El clima predominante es templado subhúmedo, aunque en menor proporción se presentan los climas templado semiseco y seco, todos con un régimen de lluvias en verano (Pavón y Meza, 2009). La temperatura media anual oscila entre los $14^{\circ} \mathrm{C}$ en la región templada y los $19^{\circ} \mathrm{C}$ en las zonas más secas (Sánchez-González, Álvarez y Tejero-Diez, 2010). La precipitación promedio anual oscila desde los $1,088 \mathrm{~mm}$ en la región húmeda a los $374 \mathrm{~mm}$ en la región seca (Pavón y Meza, 2009). La variación climática descrita anteriormente ha generado que en el PNM se encuentren distintos tipos de vegetación como bosques de Quercus, de Pinus-Quercus, de Pinus, de Juniperus (o táscate), matorral xerófilo, bosque tropical 


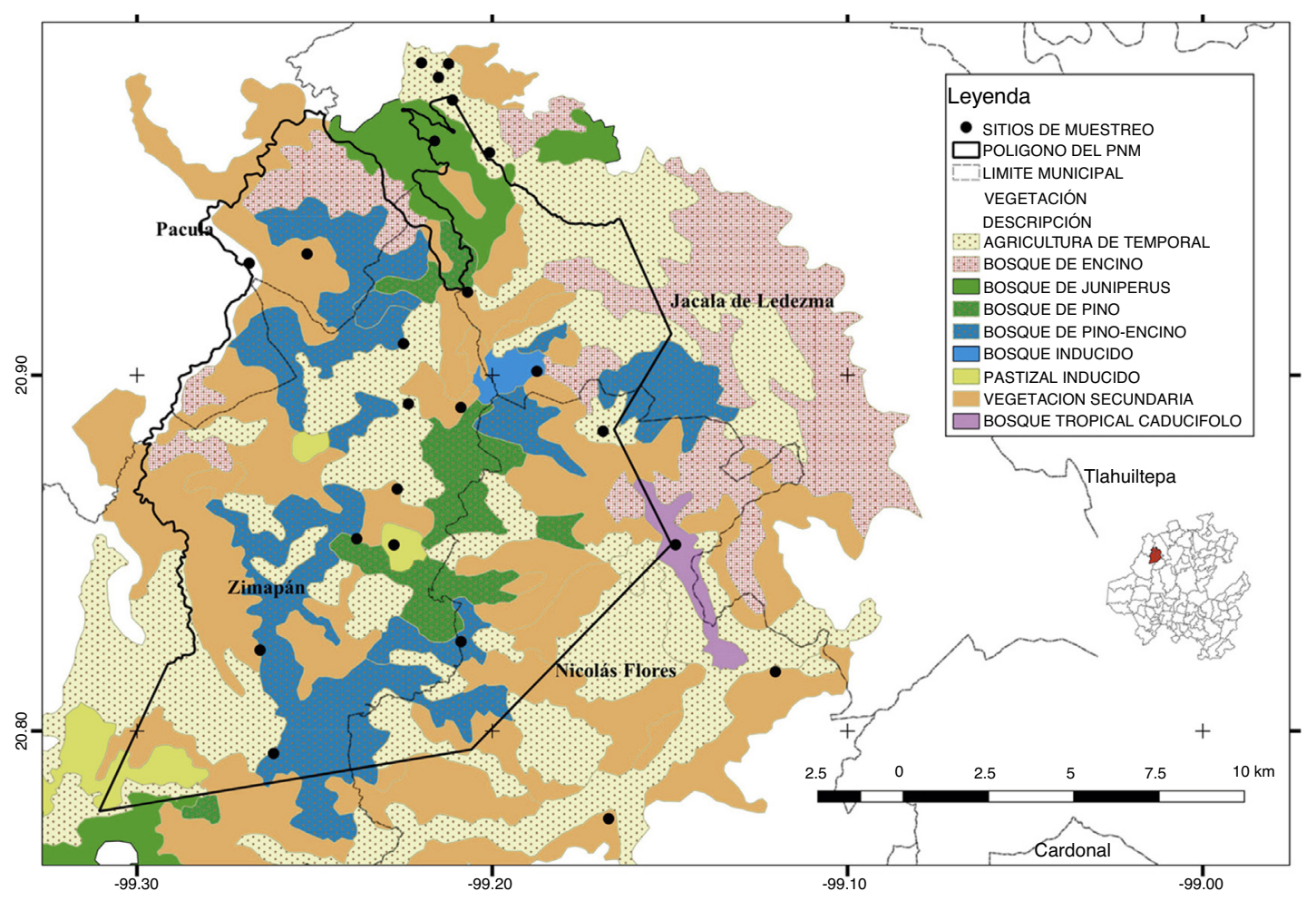

Figura 1. Mapa del Parque Nacional los Mármoles, Hidalgo, México. Con colores diferentes se indican los principales tipos de vegetación en la zona y con puntos negros los sitios de muestreo donde se establecieron transectos, puntos de conteo o estaciones de escucha. Las coordenadas usadas se muestran en formato UTM. Modificado de Inegi (2016).

caducifolio y pastizal inducido (Ramírez-Cruz, SánchezGonzález y Tejero-Diez, 2009; Randell, 2008; Rzedowski, 1978; Sánchez-González et al., 2010; fig. 1).

El bosque de Quercus en el PNM está constituido principalmente por $Q$. crassifolia, $Q$. laeta, $Q$. mexicana y $Q$. affinis. Los encinares ocupan más del $20 \%$ del territorio de esta ANP, siendo el tipo de vegetación más abundante en la región (Sánchez-González et al., 2010). Los bosques de PinusQuercus o Quercus-Pinus (que se diferencian por la dominancia numérica de uno u otro género) están constituidos por especies de Pinus con 2 o más asociaciones, principalmente bosques de $P$. cembroides con $J$. deppeana y bosques de $P$. greggii, $P$. teocote, $P$. patula y $P$. pseudostrobus con $Q$. mexicana y $Q$. crassifolia (García-Sánchez, Sánchez-González y Villaseñor, 2014; Randell, 2008, Sánchez-González et al., 2010). El bosque de Pinus está constituido por $P$. cembroides, $P$. greggii, $P$. teocote, P. patula y P. pseudostrobus (Randell, 2008). El bosque de Juniperus está dominado principalmente por J. flaccida y $J$. deppeana (Ramírez-Cruz et al., 2009; Randell, 2008; SánchezGonzález et al., 2010). El matorral xerófilo está conformado por Acacia berlandieri, Caesalpinia mexicana, Dalea bicolor, Karwinskia humboldtiana, Mimosa aculeaticarpa y Senna racemosa (Ramírez-Cruz et al., 2009). El bosque tropical caducifolio domina las zonas más bajas y cálidas del parque, siendo las especies arbóreas predominantes del género Bursera y Lysiloma, con un componente arbustivo y herbáceo muy abundante (Randell, 2008).
El registro de las aves presentes en el PNM se llevó a cabo a través de muestreos sistemáticos realizados en 2 temporadas entre 2013 y 2015 con visitas mensuales (3-4 días) y muestreos esporádicos entre 2011 y 2016 ( $c a$. 10 días por año). Durante la primera temporada de muestreo sistemático (2013-2014) se obtuvieron registros exclusivamente de colibríes (Guzmán, 2015). Para el registro de estos se visitaron bosques de Quercus y Pinus-Quercus, en los que se ubicaron 2 trayectos de $1 \mathrm{~km}$ de longitud por $40 \mathrm{~m}$ de ancho, mismos que fueron recorridos en aproximadamente $1 \mathrm{~h}$ siguiendo el método de Emlen (1971) modificado por Ortiz-Pulido y Díaz (2001) con el objetivo de tener más registros de colibríes, para lo cual se puso especial atención en agregaciones de flores cuyas características (colores llamativos, y corolas tubulares) indicaron que podrían ser polinizadas por colibríes. En la segunda temporada de muestreo sistemático (2014-2015), se visitaron 3 tipos de vegetación (bosques de Pinus, Pinus-Quercus y Juniperus). Para ello se colocaron 2 trayectos de $1 \mathrm{~km}$ de longitud por tipo de vegetación sobre los que se ubicaron 5 puntos de conteo separados entre sí cada $200 \mathrm{~m}$. Los puntos de conteo tuvieron un radio de $25 \mathrm{~m}$ dentro del cual se identificó a los individuos durante $10 \mathrm{~min}$ de observación en cada tipo de vegetación.

Los muestreos esporádicos correspondieron a registros casuales, realizados principalmente en bosque de Quercus, matorral xerófilo y bosque tropical caducifolio. En el caso de las aves con actividad nocturna (búhos y tapacaminos) se 
empleó el método de estaciones de escucha y reclamo sobre caminos de terracería con vegetación poco perturbada (sensu Valencia-Herverth et al., 2012a). En este método se contabilizan las respuestas sonoras de las aves de interés a grabaciones de diferentes especies. Este método aumenta la probabilidad de detectar la presencia de aves nocturnas durante los muestreos. Este método resulta más efectivo respecto de la observación directa, consecuencia del periodo de actividad de estos grupos de aves y su forma de comunicación en la oscuridad. Salvo para búhos y tapacaminos, las observaciones se realizaron a partir de la salida del sol hasta cerca del mediodía, periodo en que las aves diurnas están más activas (sensu Buckland, 2006). Para cada individuo observado se tomaron datos básicos como el nombre de la especie, fecha, coordenadas geográficas del sitio y el tipo de vegetación donde se observó (Ralph et al., 1996). Para la identificación de las aves se utilizaron binoculares de $10 \times 50 \mathrm{~mm}$ y $8 \times 40 \mathrm{~mm}$ y el apoyo de guías de campo especializadas (Howell y Webb, 1995; National Geographic Society, 2011; Peterson y Chalif, 2008).

La nomenclatura taxonómica usada en este trabajo es la de la American Ornithologists' Union (AOU, 1998) y suplementos adicionales (http://checklist.aou.org/). La categoría de endemismo se determinó con base en lo propuesto por GonzálezGarcía y Gómez-de Silva (2003). Los grados de vulnerabilidad se adjudicaron conforme a lo establecido en la Nom-059Semarnat-2010 (Semarnat, 2010) y lo indicado por la Unión Internacional para la Conservación de la Naturaleza (IUCN, 2016). La estacionalidad se determinó con información generada durante el estudio e información bibliográfica (Berlanga et al., 2015; Howell y Webb, 1995; Martínez-Morales et al., 2007; Ortiz-Pulido, Gómez-de Silva, González-García y Álvarez, 1995); a cada especie se les asignó alguna de las siguientes categorías: 1) residente, especie que se reproduce en la región y se puede observar durante todo el año; 2) migratoria de invierno, se reproducen en Norteamérica y pasan el invierno en México o más al Sur; 3 ) migratoria de verano, se encuentran en México durante la temporada de reproducción entre los meses de marzo y septiembre; 4) transitoria, especie que usa el área como zona de paso durante la migración al sur en otoño o al norte durante la primavera. El valor de abundancia relativa se asignó empleando el esfuerzo de muestreo estandarizado sugerido por Ortiz-Pulido y Díaz (2001); este método es una modificación al planteando por Emlen (1971), con el objetivo de hacer comparables los valores de abundancia obtenidos en trabajos similares pero que tienen un esfuerzo de muestreo diferente. En este trabajo el valor de abundancia se obtuvo al dividir el número de registros de una especie entre las horas muestreadas y multiplicando el resultado por 100. Una especie fue considerada rara si se registró menos de 3.5 veces, común si se registró entre 3.5 y 21 veces, y abundante si tuvo más de 22 registros en un esfuerzo de muestreo estandarizado. No se asignó alguna categoría cuando la naturaleza del muestreo no lo permitió (muestreos esporádicos) o no existió información precisa de los avistamientos. La riqueza por tipo de vegetación (bosque de Pinus-Quercus, bosque de Pinus, boque de Juniperus, bosque de Quercus, matorral xerófilo y bosque tropical caducifolio) se determinó considerando el sitio en que se registró a cada una de las especies durante el estudio. Se asignó un gremio trófico para cada especie con base en lo propuesto por González-Salazar, Martínez-Meyer y López-Santiago (2014), considerando que un gremio trófico se define por 3 variables: 1) la dieta principal, 2) el sustrato de forrajeo y 3) el periodo de actividad. Se empleó información bibliográfica adicional para complementar los datos por especie de las 3 variables mencionadas (Hoyo, Elliot y Sargatal, 1992, 1996, 1999, 2005; Ortiz-Pulido et al., 2010; Ramírez-Albores, 2006).

Para determinar el grado en que el listado estuvo completo se utilizaron modelos de acumulación de especies (Herzog, Kessler y Cahill, 2002). Para ello se usaron 2 grupos de datos, aquellos obtenidos solo con el muestreo sistemático (durante los años 2014 y 2015) y considerando todos los datos (tomando en cuenta los datos del muestreo sistemático más los registros casuales). Para ello se utilizó el programa EstimateS Ver. 9.1 (Colwell, 2013), en donde se usó el estimador no paramétrico Chao 1 para el muestreo sistemático, ya que la naturaleza del muestreo permitió utilizar los datos de abundancia de las especies (Magurran, 2004; Moreno, 2001); y para el análisis de todos los datos se usaron los estimadores no paramétricos, Chao 2, Jacknife 1 y bootstrap, porque con ellos se pueden usar datos de presenciaausencia y no se asume «a priori» un tipo de distribución del conjunto de datos (Moreno, 2001). Los días de muestreo sirvieron como unidad de esfuerzo y la curva se ajustó con base en 100 repeticiones aleatorias de acuerdo al orden de las muestras (Colwell y Coddington, 1994). Con los resultados obtenidos se graficó la riqueza observada y la riqueza estimada. También, se utilizó lo propuesto por Gómez-de Silva y Medellín (2001) como criterio adicional para evaluar si el inventario de especies del área de estudio estaba «completo». Ellos sugieren que una lista está incompleta cuando falta alguna de las 21 familias y 17 géneros «omnipresentes» en México.

Para la consideración de nuevos registros se aplicaron los criterios sugeridos por Sánchez-González (2013), quien hace mención de que la autenticidad de un nuevo registro debe ser válido si una especie se registra por primera vez en la entidad política o región biótica distinta. Esto fue complementado utilizando como referencia los mapas propuestos por Howell y Webb (1995) y los mapas de distribución potencial de aves de México elaborados por Navarro y Peterson (2007).

\section{Resultados}

Se registraron 20 órdenes, 47 familias y 195 especies de aves en el PNM (apéndice). Las familias mejor representadas fueron Parulidae con 21 especies, Tyrannidae (17 especies), Turdidae (11 especies), Cardinalidae y Emberizidae (ambas con 10 especies). Con base en la estacionalidad, 137 especies fueron catalogadas como residentes permanentes, 44 como migratorias invernales, 6 como migratorias de verano y 8 como transitorias. Diecisiete especies de aves resultaron abundantes (Cathartes aura, Fulica americana, Columba livia, Eugenes fulgens, Hylocharis leucotis, Melanerpes formicivorus, Vireo huttoni, Aphelocoma wollweberi, Myadestes occidentalis, Setophaga townsendi, Basileuterus rufifrons, Pipilo maculatus, Melozone fusca, Junco phaeonotus, Piranga flava, Cardinalis 


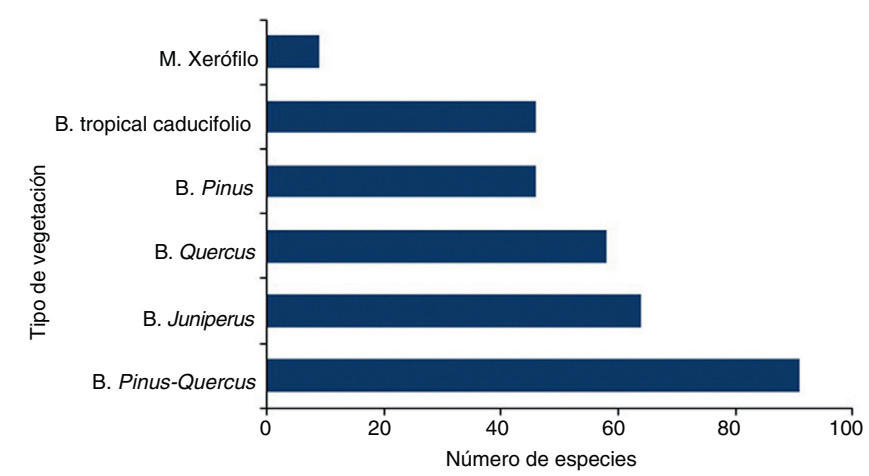

Figura 2. Riqueza de especies de aves por tipo de vegetación en el Parque Nacional Los Mármoles, Hidalgo, México.

cardinalis y Haemorhous mexicanus), 60 comunes y 118 raras (apéndice).

El mayor número de especies de aves se registró en el bosque de Pinus-Quercus (91 especies), seguido por el bosque de Juniperus (64), el bosque de Quercus (57), el bosque de Pinus y el bosque tropical caducifolio (ambas con 46 especies) y el matorral xerófilo (9; fig. 2). En el PNM se registraron aves de 21 gremios tróficos. Tres de ellos, el de insectívoro de follaje debajo del dosel, el de insectívoro en el aire debajo del dosel y el de granívoro de suelo, presentaron más especies $(31,24$ y 23 respectivamente; fig. 3).

De acuerdo con los estimadores no paramétricos se registró entre el $72 \%$ y el $85 \%$ de las especies de aves presentes en el PNM. Ciento tres especies fueron observadas usando el muestreo sistemático, calculándose con esos datos una riqueza esperada de 136 especies para los tipos de vegetación muestreados con ese método (se registró el 75\% de las especies esperadas; fig. 4). Considerando todos los datos (195 especies), usando el estimador Chao 2 se esperan 267 especies, mientras que con el estimador Jacknife 1 se esperan 270 especies (para ambos casos cerca del $72 \%$ de las especies registradas); en cambio se estimaron 228 especies con el estimador bootstrap (85\% de las especies registradas; fig. 5).

Las curvas de acumulación de especies para los 2 tipos de datos (sistemáticos y todos los datos) no se estabilizaron (figs. 4 y 5). Además, se registraron 21 familias y 14 géneros omnipresentes en México, estando ausentes 3 géneros (Riparia, Petrochelidon y Progne) de la familia Hirundidae.

Del total de las especies registradas en el PNM, el 20.5\% se encuentran bajo alguna categoría de riesgo a nivel nacional y/o nivel internacional, o son especies endémicas. De este porcentaje 11 especies de aves registradas se encuentran bajo alguna categoría de riesgo según la legislación mexicana. Dentro de la categoría amenazadas se incluyen Aquila chrysaetos, Falco femoralis, Psittacara holochlorus, Aphelocoma unicolor, Myadestes unicolor y Turdus infuscatus, y en sujetas a protección especial se incluyen a Cyrtonyx montezumae, Chondrohierax uncinatus, Buteo swainsoni, Falco peregrinus y Myadestes occidentalis. A nivel internacional, Colinus virginianus, Contopus cooperi, Vireo bellii y Haemorhous cassinii, se ubican en la categoría de casi amenazadas. En cuanto a las especies endémicas a México, hay 26 especies de aves en el PNM: 8 son endémicas, 11 semiendémicas y 7 cuasiendémicas (apéndice).

\section{Discusión}

En este estudio encontramos que en el PNM habitan al menos 195 especies. Esto representa el 17\% de la avifauna nacional (Navarro-Sigüenza et al., 2014; Berlanga et al., 2015) y el $38 \%$ de la avifauna presente en el estado de Hidalgo (García y Ortiz-Pulido, 2007; Guzmán, 2015; Martínez-Morales et al., 2007, 2013; Ortiz-Pulido et al., 2010; Pineda-López, Arellano-Sanaphre y la Vega-Aguirre, 2013; Valencia-Herverth, Valencia-Herverth, Mendoza-Quijano, Linarte y Hernández, 2008; Valencia-Herverth, Valencia-Herverth y MendozaQuijano, 2010; Valencia-Herverth, Valencia-Herverth, Mendiola-González, Sánchez-Cabrera y Martínez-Morales, 2011; Valencia-Herverth et al., 2012a; Valencia-Herverth et al., 2012b; Valencia-Herverth y Valencia-Herverth, 2008).

Creemos que la diversidad de aves registrada en el PNM es debida a que en el PNM confluyen 3 provincias fisiográficas (la Sierra Madre Oriental, el Sistema Volcánico Transversal y, aunque en menor proporción, la Llanura Costera del Golfo de México; Martínez-Morales et al., 2007), lo que da origen a la presencia de aves de distintas regiones de México. Además, debido a su ubicación geográfica (centro este de México) y su topografía esta riqueza se incrementa. Un ejemplo de ello es que existen aves representativas de la vertiente del golfo, como Ortalis vetula, Psittacara holochlorus, Baeolophus atricristatus, Turdus grayi y Tiaris olivaceus; de la vertiente del Pacífico, como Amazilia violiceps y Myiarchus nuttingi; de las regiones montañosas, como Aegolius acadicus, Colibri thalasinus, Trogon mexicanus, Aphelocoma unicolor, Myadestes occidentalis y Loxia curvirostra, y del Altiplano Mexicano, como Aquila chrysaetos, Geococcyx californianus, Aphelocoma wollweberi y Toxostoma curvirostre.

De los ambientes descritos en el PNM, los templados presentan mayor riqueza de especies de aves (fig. 2) que los cálidos. Sin embargo, es posible que los ambientes más cálidos (matorral xerófilo y el bosque tropical caducifolio) presenten una mayor riqueza. Esto es posible porque en estos últimos tipos de vegetación solo se realizaron registros casuales durante este estudio y posiblemente la riqueza de aves para estos tipos de vegetación esté subestimada, particularmente porque con menor número de muestreos el bosque tropical caducifolio presenta una riqueza en aves (46 especies) igual al bosque de Pinus, siendo que este último fue muestreado durante un año. Por ello se sugiere se realicen más muestreos avifaunísticos, sobre todo en las zonas cálidas, como el bosque tropical caducifolio (en las localidades del municipio de Nicolás Flores, al Este del PNM a una altitud cercana a los $900 \mathrm{~m}$ snm), matorral xerófilo (en localidades del municipio del Jacala, al Noreste del PNM a una altitud cercana a los 2,000 m snm), y puntos aún no explorados como la barranca San Vicente, la cual nace en los cañones del centro $(2,300 \mathrm{~m} \mathrm{snm})$ y recorre el borde oeste 


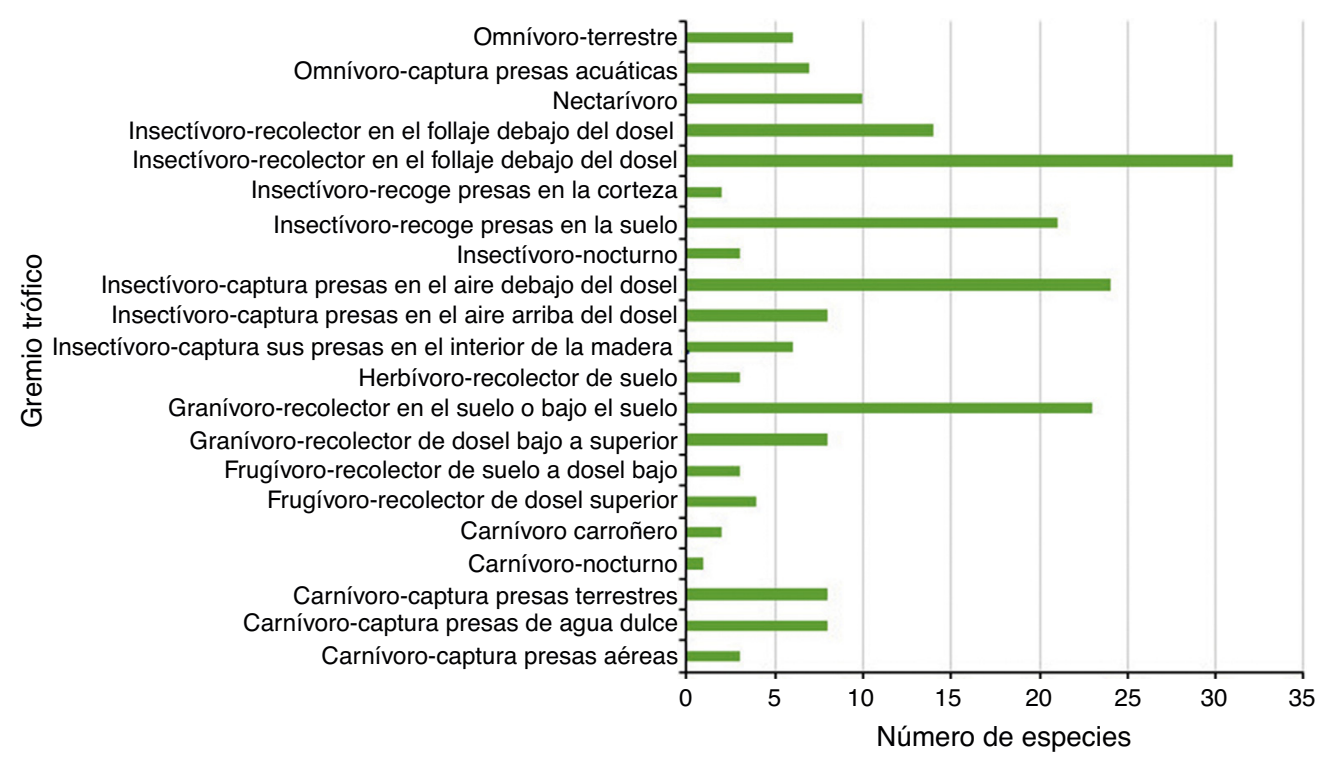

Figura 3. Riqueza de especies de aves por gremio trófico en el Parque Nacional Los Mármoles, Hidalgo, México.

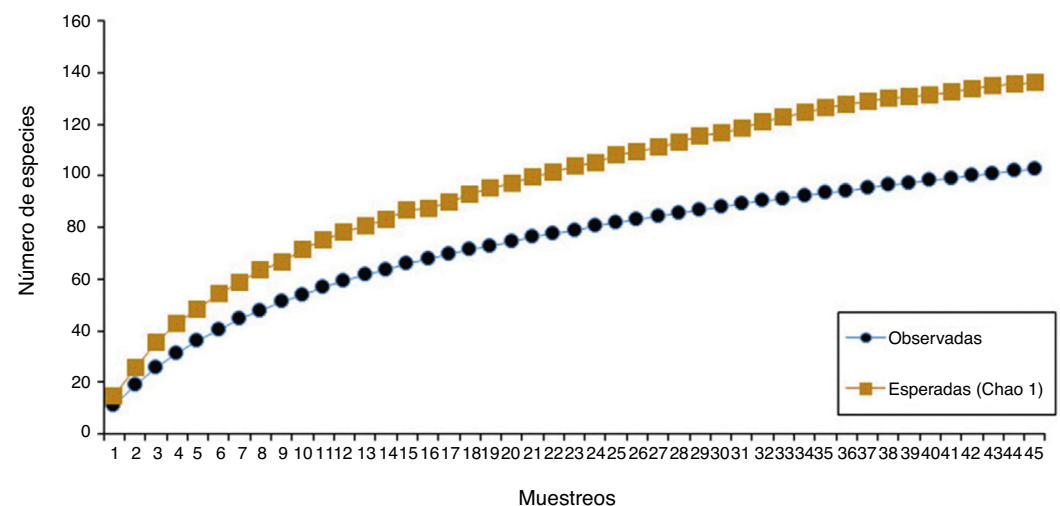

Figura 4. Curvas de acumulación de especies de aves usando datos de un muestreo estandarizado realizado en el Parque Nacional Los Mármoles, Hidalgo, México. Se presentan datos de especies observadas y esperadas (usando el estimador no paramétrico Chao 1) para los tipos de vegetación muestreados.

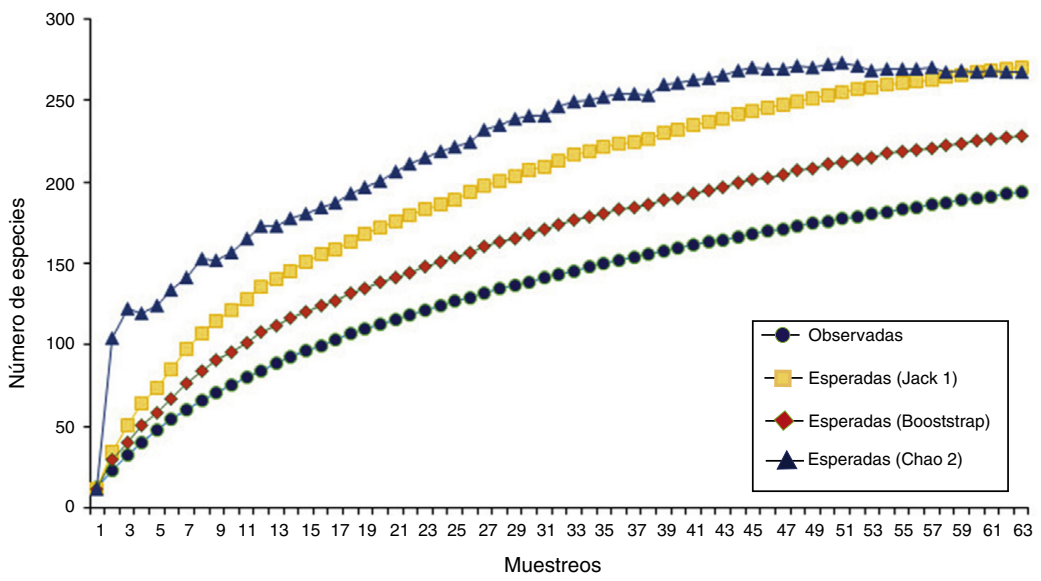

Figura 5. Curvas acumulación de especies de aves para el Parque Nacional Los Mármoles usando todos los registros obtenidos en este estudio. Se presentan datos de especies observadas y esperadas a partir de 3 modelos de acumulación de especies (Jacknife 1, Chao 2 y bootstrap). 
del PNM hasta su zona norte. Esta barranca podría contener una alta riqueza de aves ya que tiene una profundidad de $600 \mathrm{~m}$ y una longitud aproximada de $16 \mathrm{~km}$, con bosque de Quercus y matorral xerófilo como tipos de vegetación principales.

A nivel estatal, el PNM es la segunda ANP con el mayor número de aves registradas después de la RBBM en donde se han registrado 294 especies (Conanp, 2013; Ortiz-Pulido et al., 2010; Valencia-Herverth et al., 2011). En ambas ANP se distribuyen distintos tipos de vegetación, lo que puede estar determinando la diversidad de aves presente en cada sitio. Las diferencias en riqueza pueden deberse a que el PNM está dominado por bosques de coníferas y de encinos, mientras que la vegetación en la RBBM es más diversa en cuanto a tipos de vegetación; además esta última tiene mayor área $(96,042.94$ ha) que el PNM (23,150 ha). A pesar de ello, los estimadores no paramétricos de acumulación de especies obtenidos para el PNM (fig. 5) predicen una riqueza semejante a la encontrada en la RBBM, pero el inventario en el PNM es incompleto y será necesario aumentar el trabajo de campo en varias zonas de esta ANP.

Como resultado colateral de este estudio, se presenta un nuevo registro estatal, el del gorrión de Cassinii (Haemorhous cassinii), el cual se ha registrado previamente en la Reserva de la Biosfera Sierra Gorda, Querétaro (Carabias, Provencio, de la Maza y Ruiz, 1999). Además, se presentan registros relevantes de 4 especies (Ortalis vetula, Trogon elegans, Falco rufigularis y Megarynchus pitangua) en el PNM, estas especies solo se habían registrado en las zonas tropicales ubicadas al noreste de Hidalgo (Rojas-Soto, Sánchez-González y López de Aquino, 2002; Valencia-Herverth et al., 2008).

Tomando en cuenta todo lo anterior, proponemos que el PNM sea considerado en el futuro como un área de importancia para la conservación de las aves en México (Arizmendi y Márquez-Valdelamar, 1999; BirdLife, 2016; Conabio, 2002). Esto es porque presenta 26 especies endémicas, 11 especies que se encuentran en algún grado de riesgo a nivel nacional y 4 más se encuentran en peligro globalmente (Psittacara holochlorus). Además, presenta el $38 \%$ de las especies de aves de Hidalgo, el $17 \%$ de las aves habitando México y se ubica a solo $20 \mathrm{~km}$ de distancia del área de importancia para la conservación de las aves C-06-Reserva de la Biosfera Sierra Gorda, que en términos de la vagilidad de las aves, es una distancia relativamente corta.

Considerando la diversidad avifaunística del PNM creemos que es conveniente fortalecer en este las acciones de conservación de aves. Esto es porque a pesar de que esta ANP se encuentra catalogada como Parque Nacional (donde no se puede hacer actividades extractivas), en él se desarrollan acciones que pueden afectar a la avifauna presente. Por ejemplo, dentro del parque se encuentran diferentes asentamientos humanos y se llevan a cabo actividades agrícolas, ganaderas (extensiva) y mineras (extracción de mármol) (Randell, 2008). Además, varias zonas presentan pérdida de cobertura forestal por erosión y degradación de suelos. Si se llevan a cabo acciones de conservación de aves en el PNM estas se deben enfocar en especies prioritarias para la conservación, como las especies endémicas o las que tengan alguna categoría de riesgo a nivel nacional o internacional.

En síntesis, en este trabajo reportamos 195 especies de aves en el PNM, un $20.5 \%$ de las cuales se encuentran en alguna categoría de riesgo o endemismo. La riqueza del PNM representa un alto porcentaje de la diversidad avifaunística de Hidalgo. Esta información debería ser considerada por quienes elaboren acciones de conservación, educación ambiental y manejo de los recursos naturales en el PNM y áreas adyacentes.

\section{Agradecimientos}

A la Comisión Nacional de Áreas Naturales Protegidas (Conanp), a través la dirección del PNM en especial a Marco Antonio Soto, Alejandro López Portillo, Jorge Iván Ángeles Escudero y Juan Carlos García Loperena por las facilidades otorgadas para realizar este trabajo. A Cornelio Rojo, brigadista del PNM por su apoyo logístico en las salidas de campo. A Conacyt y a la Universidad Autónoma del Estado de Hidalgo (proyectos 191908, UAEH-DI-ICBI-BI-SF-008 e ICBI-PAI29), por su apoyo a esta investigación. Al Dr. William Scott Monks Sheets por su ayuda en la elaboración del resumen en inglés.
Apéndice. Especies de aves presentes en el Parque Nacional Los Mármoles, Hidalgo, México. Se indica para cada especie hábitat, alimentación, abundancia relativa, estacionalidad, estatus de riesgo para México y el mundo y endemismo para el país. Estacionalidad: R, residente permanente; $\mathrm{MI}$, migratoria de invierno; $\mathrm{MV}$, migratoria de verano; $T$, transitoria. Hábitat: $B E$, bosque de encino; BP, bosque de pino; BPE, bosque de pino-encino; $\mathrm{BJ}$, bosque de táscate; $\mathrm{SBC}$, bosque tropical caducifolio; MX, matorral xerófilo. Abundancia relativa; $\mathrm{Ab}$, abundante; $\mathbf{C m}$, común; $\mathrm{Rr}$, rara. Estatus de protección NOM-059 o IUCN: A, amenazada; Pr, protección especial; NT, casi amenazado. Endemismo: $\mathrm{CE}$, cuasiendémico; $\mathrm{E}$, endémico; $\mathrm{SE}$, semiendémico. $\mathrm{El}$ orden de las especies sigue la sugerencia de AOU (1998) y suplementos. 


\begin{tabular}{llllll}
\hline Orden & Estacionalidad & Hábitat & Abundancia NOM-059 IUCN Endemismo Gremio \\
Familia & & & & \\
Especie & & & & \\
\hline
\end{tabular}

Anseriformes

Anatidae

$\begin{array}{lll}\text { Anas platyrhynchos } & \mathrm{R} & \mathrm{BE} \\ \text { Anas discors } & \mathrm{MI} & \mathrm{BE} \\ \text { Anas clypeata } & \mathrm{MI} & \mathrm{BE} \\ \text { Anas cyanoptera } & \mathrm{R} & \mathrm{MX} \\ \text { Anas acuta } & \mathrm{MI} & \mathrm{BE} \\ \text { Anas crecca } & \mathrm{MI} & \mathrm{BE} \\ \text { Oxyura jamaicensis } & \mathrm{R} & \mathrm{MX}, \mathrm{BE}\end{array}$

Omnívoro - captura presas acuáticas Omnívoro - captura presas acuáticas Omnívoro - captura presas acuáticas Omnívoro - captura presas acuáticas Herbívoro - recolector de suelo Herbívoro - recolector de suelo Herbívoro - recolector de suelo

Galliformes

Cracidae

$\begin{array}{lll}\text { Ortalis vetula } & \mathrm{R} & \mathrm{BE}\end{array}$

Odontophoridae

Colinus virginianus

$\mathrm{R}$

BPE

NT

Cyrtonyx montezumae

$\mathrm{R}$

BPE

$\operatorname{Pr}$

Podicipediformes

Podicipedidae

Podilymbus podiceps

$\mathrm{R}$

$\mathrm{BE}$

Omnívoro - captura presas acuáticas

Columbiformes

Columbidae

Columba livia

$\mathrm{R}$

$\mathrm{BE}$

Patagioenas fasciata

$\mathrm{R}$

BE

Columbina inca

$\mathrm{R}$

BJ, BP, SBC

$\mathrm{Cm}$

Columbina passerina

$\mathrm{R}$

BE, SBC

Leptotila verreauxi

$\mathrm{R}$

BPE

Zenaida asiatica

$\mathrm{R}$

SBC

Zenaida macroura

$\mathrm{R}$

BJ, SBC

$\mathrm{Cm}$

Cuculiformes

Cuculidae

Geococcyx californianus

$\mathrm{R}$

BPE, BJ

Frugívoro - recolector de suelo a dosel bajo

Granívoro - recolector en el suelo o bajo el suelo

Granívoro - recolector en el suelo o bajo el suelo

Caprimulgiformes

Caprimulgidae

Antrostomus vociferus $\quad$ MI $\quad \mathrm{BE}$

$\begin{array}{lll}\text { Antrostomus arizonae } & \mathrm{R} & \mathrm{BE}\end{array}$

Apodiformes

Apodidae

Streptoprocne zonaris

$\mathrm{R}$

$\mathrm{BE}$

Chaetura vauxi

$\mathrm{T}$

BE

Aeronautes saxatalis

$\mathrm{R}$

BPE

Trochilidae

Colibri thalassinus

Eugenes fulgens

Lampornis clemenciae

Calothorax lucifer

Archilochus colubris

Cynanthus latirostris

Selasphorus platycercus

$\begin{array}{lll}\text { R } & \text { SBC } & \\ \text { R } & \text { BJ, BPE, BP, BE } & \text { Ab } \\ \text { R } & \text { BPE, SBC } & \text { Cm } \\ \text { MV } & \text { MX } & \\ \text { T } & \text { BJ, BP } & \mathrm{Cm} \\ \text { R } & \text { BJ } & \text { Cm } \\ \text { MV } & \text { BJ } & \text { Rr }\end{array}$

SBC

Granívoro - recolector en el suelo o bajo el suelo

Frugívoro - recolector de suelo a dosel bajo

Granívoro - recolector en el suelo o bajo el suelo

Granívoro - recolector en el suelo o bajo el suelo

Granívoro - recolector en el suelo o bajo el suelo

Granívoro - recolector en el suelo o bajo el suelo

Granívoro - recolector en el suelo o bajo el suelo

Carnívoro - captura presas terrestres

Insectívoro - nocturno

Insectívoro - nocturno

Insectívoro - captura presas en el aire arriba del dosel

Insectívoro - captura presas en el aire arriba del dosel

Insectívoro - captura presas en el aire arriba del dosel

Nectarívoro

Nectarívoro

$\begin{array}{ll}\text { SE } & \text { Nectarívoro } \\ \text { SE } & \text { Nectarívoro }\end{array}$

Nectarívoro

Nectarívoro

Nectarívoro 


\begin{tabular}{lllllll}
\hline Orden & Estacionalidad & Hábitat & Abundancia NOM-059 IUCN & Endemismo Gremio
\end{tabular}

Familia

Especie

Amazilia violiceps

Hylocharis leucotis

Gruiformes

Rallidae

Fulica americana

Charadriiformes

Recurvirostridae

Himantopus mexicanus

$\mathrm{R}$

MX

Charadriidae

Charadrius vociferus

$\mathrm{R}$

BE

Scolopaidae

Gallinago gallinago

MI

BJ

Suliformes

Phalacrocoracidae

Phalacrocorax brasilianus

R

BE

Pelecaniformes

Ardeidae

Ardea herodias

Egretta thula

Egretta caerulea

Bubulcus ibis

Nycticorax nycticorax

MI

$\mathrm{R}$

$\mathrm{R}$

Cathartes aura

Accipitriformes

Accipitridae

Chondrohierax uncinatus

Circus cyaneus

Buteo plagiatus

Buteo swainsoni

Buteo jamaicensis

Aquila chrysaetos

Strigiformes

Strigidae

Megascops trichopsis

Glaucidium gnoma

Aegolius acadicus

Trogoniformes

Trogonidae

Trogon elegans

Trogon mexicanus

Coraciiformes

Alcedinidae

Chloroceryle americana

SBC

MX
BJ, BPE, BP, SBC $\mathrm{Ab}$

Omnívoro - captura presas acuáticas

Carnívoro - captura presas de agua dulce

Insectívoro - recoge presas en el suelo

Omnívoro - captura presas acuáticas

Carnívoro - captura presas de agua dulce

Carnívoro - captura presas de agua dulce

Carnívoro - captura presas de agua dulce

Carnívoro - captura presas de agua dulce

Carnívoro - captura presas de agua dulce

Carnívoro - captura presas de agua dulce

Carnívoro carroñero

$\mathrm{BJ}, \mathrm{BPE}, \mathrm{BP}, \mathrm{BE} \quad \mathrm{Cm}$

$\mathrm{BJ}, \mathrm{BPE}, \mathrm{BP}, \mathrm{BE} \quad \mathrm{Ab}$

Carnívoro carroñero

Carnívoro - captura presas terrestres Carnívoro - captura presas terrestres Carnívoro - captura presas terrestres Carnívoro - captura presas terrestres Carnívoro - captura presas terrestres Carnívoro - captura presas terrestres

Insectívoro - nocturno

Carnívoro - captura presas terrestres Carnívoro - nocturno

Frugívoro - recolector de dosel superior

Frugívoro - recolector de dosel superior

Carnívoro - captura presas de agua dulce 


\begin{tabular}{lllllll}
\hline Orden & Estacionalidad & Hábitat & Abundancia NOM-059 IUCN Endemismo Gremio \\
Familia & & & & \\
Especie & & & &
\end{tabular}

Piciformes

Picidae

Melanerpes formicivorus

BJ, BPE, BP, BE $\quad \mathrm{Ab}$

Melanerpes aurifrons $\quad \mathrm{R}$

SBC

Sphyrapicus varius

MI

BJ

$\operatorname{Rr}$

Picoides scalaris

$\mathrm{R}$

BJ, BPE, SBC

$\mathrm{Rr}$

Picoides villosus

$\mathrm{R}$

BJ, BPE

$\mathrm{Cm}$

Colaptes auratus

$\mathrm{R}$

BPE

$\mathrm{Rr}$

Falconiformes

Falconidae

Falco sparverius

Falco femoralis

Falco rufigularis

Falco peregrinus

Psittaciformes

Psittacidae

Psittacara holochlorus

$\mathrm{R}$

SBC

BPE, SBC

$\mathrm{R}$

$\mathrm{R}$

$\mathrm{R}$

MI

$\mathrm{BE}$

BE

A

BPE

$\mathrm{Rr}$

$\operatorname{Pr}$

A

Passeriformes

Tyrannidae

Mitrephanes phaeocercus

Contopus cooperi

Contopus pertinax

$\mathrm{R}$

Contopus sordidulus

MV

BE

Empidonax albigularis

MV

Empidonax hammondii

MI

$\mathrm{R}$

$\mathrm{R}$

BPE, SBC

NT

Empidonax affinis

Sayornis nigricans

Sayornis phoebe

Pyrocephalus rubinus

MI

BJ, BPE, BP, SBC $\quad \mathrm{Cm}$

Myiarchus tuberculifer

$\mathrm{R}$

$\mathrm{R}$

Myiarchus cinerascens

MI

Myiarchus crinitus

$\mathrm{T}$

Myiarchus nuttingi

$\mathrm{R}$

Megarynchus pitangua

$\mathrm{R}$

$\mathrm{R}$

$\mathrm{SBC}$

Myiozetetes similis

$\mathrm{R}$

Tyrannus vociferans
E

Insectívoras - capturan sus presas en el interior de la madera Insectívoras - capturan sus presas en el interior de la madera Insectívoras - capturan sus presas en el interior de la madera Insectívoras - capturan sus presas en el interior de la madera Insectívoras - capturan sus presas en el interior de la madera Insectívoras - capturan sus presas en el interior de la madera

Insectívoro - recoge presas en el suelo

Carnívoro - captura presas aéreas Carnívoro - captura presas aéreas

Carnívoro - captura presas aéreas

Frugívoro - recolector de dosel superior

Insectívoro - captura presas en el aire debajo del dosel Insectívoro - captura presas en el aire debajo del dosel Insectívoro - captura presas en el aire debajo del dosel Insectívoro - captura presas en el aire debajo del dosel

Insectívoro - captura presas en el aire debajo del dosel Insectívoro - captura presas en el aire debajo del dosel Insectívoro - captura presas en el aire debajo del dosel Insectívoro - captura presas en el aire debajo del dosel Insectívoro - captura presas en el aire debajo del dosel

Insectívoro - captura presas en el aire debajo del dosel Insectívoro - captura presas en el aire debajo del dosel Insectívoro - captura presas en el aire debajo del dosel Insectívoro - captura presas en el aire debajo del dosel Insectívoro - captura presas en el aire debajo del dosel Insectívoro - captura presas en el aire debajo del dosel Insectívoro - captura presas en el aire debajo del dosel insoctivoro - captura presas en el aire debajo del dosel 


\begin{tabular}{|c|c|c|c|c|c|c|c|}
\hline $\begin{array}{l}\text { Orden } \\
\text { Familia } \\
\text { Especie }\end{array}$ & Estacionalidad & Hábitat & Abundancia & NOM-059 & IUCN & Endemismo & Gremio \\
\hline \multicolumn{8}{|l|}{ Tityridae } \\
\hline Pachyramphus major & $\mathrm{R}$ & $\mathrm{BP}$ & $\mathrm{Rr}$ & & & & $\begin{array}{l}\text { Insectívoro - captura presas en el } \\
\text { aire debajo del dosel }\end{array}$ \\
\hline Pachyramphus aglaiae & $\mathrm{R}$ & BJ & $\mathrm{Rr}$ & & & & $\begin{array}{l}\text { Insectívoro - captura presas en el } \\
\text { aire debajo del dosel }\end{array}$ \\
\hline \multicolumn{8}{|l|}{ Vireonidae } \\
\hline Vireo bellii & MI & $\mathrm{BE}$ & & & NT & & $\begin{array}{l}\text { Insectívoro - recolector en el follaje } \\
\text { de bajo del dosel }\end{array}$ \\
\hline Vireo huttoni & $\mathrm{R}$ & BJ, BPE, BP & $\mathrm{Ab}$ & & & & $\begin{array}{l}\text { Insectívoro - recolector en el follaje } \\
\text { de bajo del dosel }\end{array}$ \\
\hline Vireo cassinii & MI & $\mathrm{BE}$ & & & & & $\begin{array}{l}\text { Insectívoro - recolector en el follaje } \\
\text { de bajo del dosel }\end{array}$ \\
\hline Vireo gilvus & MI & $\mathrm{BPE}$ & & & & & $\begin{array}{l}\text { Insectívoro - recolector en el follaje } \\
\text { de bajo del dosel }\end{array}$ \\
\hline \multicolumn{8}{|l|}{ Corvidae } \\
\hline Cyanocitta stelleri & $\mathrm{R}$ & $\mathrm{BE}$ & & & & & Omnívoro - terrestre \\
\hline Aphelocoma wollweberi & $\mathrm{R}$ & BJ, BPE, BP & $\mathrm{Ab}$ & & & & Omnívoro - terrestre \\
\hline Aphelocoma unicolor & $\mathrm{R}$ & $\mathrm{BJ}, \mathrm{BPE}$ & $\mathrm{Cm}$ & A & & & Omnívoro - terrestre \\
\hline Corvus cryptoleucus & MI & BPE & & & & & Omnívoro - terrestre \\
\hline Corvus corax & $\mathrm{R}$ & $\mathrm{BJ}, \mathrm{BPE}$ & $\mathrm{Rr}$ & & & & Omnívoro - terrestre \\
\hline \multicolumn{8}{|l|}{ Hirundinidae } \\
\hline Tachycineta bicolor & MI & BPE & & & & & $\begin{array}{l}\text { Insectívoro - captura presas en el } \\
\text { aire arriba del dosel }\end{array}$ \\
\hline Tachycineta thalassina & $\mathrm{R}$ & BPE, BE & $\mathrm{Cm}$ & & & & $\begin{array}{l}\text { Insectívoro - captura presas en el } \\
\text { aire arriba del dosel }\end{array}$ \\
\hline Stelgidopteryx serripennis & $\mathrm{R}$ & BPE & & & & & $\begin{array}{l}\text { Insectívoro - captura presas en el } \\
\text { aire arriba del dosel }\end{array}$ \\
\hline Hirundo rustica & MV & BJ, BPE, BP & $\mathrm{Cm}$ & & & & $\begin{array}{l}\text { Insectívoro - captura presas en el } \\
\text { aire arriba del dosel }\end{array}$ \\
\hline \multicolumn{8}{|l|}{ Paridae } \\
\hline Baeolophus wollweberi & $\mathrm{R}$ & $\mathrm{BJ}, \mathrm{BP}$ & $\mathrm{Cm}$ & & & & $\begin{array}{l}\text { Insectívoro - recolector en el follaje } \\
\text { de bajo del dosel }\end{array}$ \\
\hline Baeolophus atricristatus & $\mathrm{R}$ & BJ, BP, SBC & $\mathrm{Rr}$ & & & & $\begin{array}{l}\text { Insectívoro - recolector en el follaje } \\
\text { de bajo del dosel }\end{array}$ \\
\hline \multicolumn{8}{|l|}{ Remizidae } \\
\hline Auriparus flaviceps & $\mathrm{R}$ & MX & & & & & $\begin{array}{l}\text { Insectívoro - recolector en el follaje } \\
\text { de bajo del dosel }\end{array}$ \\
\hline \multicolumn{8}{|l|}{ Aegithalidae } \\
\hline Psaltriparus minimus & $\mathrm{R}$ & $\mathrm{BJ}, \mathrm{BPE}$ & $\mathrm{Cm}$ & & & & $\begin{array}{l}\text { Insectívoro - recolector en el follaje } \\
\text { de bajo del dosel }\end{array}$ \\
\hline \multicolumn{8}{|l|}{ Certhiidae } \\
\hline Certhia americana & $\mathrm{R}$ & BPE & $\mathrm{Rr}$ & & & & $\begin{array}{l}\text { Insectívoro - recoge presas en la } \\
\text { corteza }\end{array}$ \\
\hline \multicolumn{8}{|l|}{ Troglodytidae } \\
\hline Salpinctes obsoletus & $\mathrm{R}$ & BJ, BPE & $\mathrm{Cm}$ & & & & $\begin{array}{l}\text { Insectívoro - recoge presas en el } \\
\text { suelo }\end{array}$ \\
\hline Catherpes mexicanus & $\mathrm{R}$ & $\mathrm{BJ}, \mathrm{BPE}, \mathrm{SBC}$ & $\mathrm{Cm}$ & & & & $\begin{array}{l}\text { Insectívoro - recoge presas en el } \\
\text { suelo }\end{array}$ \\
\hline Troglodytes aedon & $\mathrm{R}$ & $\mathrm{BE}$ & $\mathrm{Rr}$ & & & & $\begin{array}{l}\text { Insectívoro - recolector en el follaje } \\
\text { de bajo del dosel }\end{array}$ \\
\hline Thryothorus ludovicianus & $\mathrm{T}$ & BPE & $\mathrm{Rr}$ & & & & $\begin{array}{l}\text { Insectívoro - recoge presas en el } \\
\text { suelo }\end{array}$ \\
\hline Thryomanes bewickii & $\mathrm{R}$ & BJ, BPE, SBC & $\mathrm{Cm}$ & & & & $\begin{array}{l}\text { Insectívoro - recoge presas en el } \\
\text { suelo }\end{array}$ \\
\hline Campylorhynchus gularis & $\mathrm{R}$ & BJ, BP, SBC & $\mathrm{Cm}$ & & & $\mathrm{E}$ & $\begin{array}{l}\text { Insectívoro - recoge presas en el } \\
\text { suelo }\end{array}$ \\
\hline $\begin{array}{l}\text { Campylorhynchus } \\
\text { brunneicapillus }\end{array}$ & $\mathrm{R}$ & BJ,BPE, SBC & $\mathrm{Rr}$ & & & & $\begin{array}{l}\text { Insectívoro - recoge presas en el } \\
\text { suelo }\end{array}$ \\
\hline Henicorhina leucophrys & $\mathrm{R}$ & BPE, BE & $\mathrm{Rr}$ & & & & $\begin{array}{l}\text { Insectívoro - recoge presas en el } \\
\text { suelo }\end{array}$ \\
\hline \multicolumn{8}{|l|}{ Polioptilidae } \\
\hline Polioptila caerulea & $\mathrm{R}$ & $\mathrm{BE}, \mathrm{SBC}$ & & & & & $\begin{array}{l}\text { Insectívoro - recolector en el follaje } \\
\text { de bajo del dosel }\end{array}$ \\
\hline
\end{tabular}




\begin{tabular}{|c|c|c|c|c|c|c|c|}
\hline $\begin{array}{l}\text { Orden } \\
\text { Familia } \\
\text { Especie }\end{array}$ & Estacionalidad & Hábitat & Abundancia & NOM-059 & IUCN & Endemismo & Gremio \\
\hline Polioptila melanura & $\mathrm{R}$ & BJ & $\mathrm{Rr}$ & & & & $\begin{array}{l}\text { Insectívoro - recolector en el follaje } \\
\text { de bajo del dosel }\end{array}$ \\
\hline \multicolumn{8}{|l|}{ Regulidae } \\
\hline Regulus calendula & MI & $\mathrm{BPE}, \mathrm{BE}$ & $\mathrm{Cm}$ & & & & $\begin{array}{l}\text { Insectívoro - recolector en el follaje } \\
\text { de bajo del dosel }\end{array}$ \\
\hline \multicolumn{8}{|l|}{ Turdidae } \\
\hline Sialia sialis & $\mathrm{R}$ & $\mathrm{BP}$ & $\mathrm{Rr}$ & & & & $\begin{array}{l}\text { Insectívoro - captura presas en el } \\
\text { aire debajo del dosel }\end{array}$ \\
\hline Sialia mexicana & $\mathrm{R}$ & $\mathrm{BP}$ & $\mathrm{Cm}$ & & & & $\begin{array}{l}\text { Insectívoro - captura presas en el } \\
\text { aire debajo del dosel }\end{array}$ \\
\hline Myadestes occidentalis & $\mathrm{R}$ & BJ, BPE, BP, BE & $\mathrm{Ab}$ & $\operatorname{Pr}$ & & & $\begin{array}{l}\text { Insectívoro - captura presas en el } \\
\text { aire debajo del dosel }\end{array}$ \\
\hline Myadestes unicolor & $\mathrm{R}$ & $\mathrm{BE}$ & & A & & & $\begin{array}{l}\text { Insectívoro - captura presas en el } \\
\text { aire debajo del dosel }\end{array}$ \\
\hline Catharus aurantiirostris & $\mathrm{R}$ & BJ & $\mathrm{Rr}$ & & & & $\begin{array}{l}\text { Insectívoro - recoge presas en el } \\
\text { suelo }\end{array}$ \\
\hline Catharus occidentalis & $\mathrm{R}$ & BPE & & & & $\mathrm{E}$ & $\begin{array}{l}\text { Insectívoro - recoge presas en el } \\
\text { suelo }\end{array}$ \\
\hline Catharus guttatus & MI & BPE & $\operatorname{Rr}$ & & & & $\begin{array}{l}\text { Insectívoro - recoge presas en el } \\
\text { suelo }\end{array}$ \\
\hline Turdus infuscatus & $\mathrm{R}$ & BPE & $\mathrm{Rr}$ & A & & & $\begin{array}{l}\text { Insectívoro - recoge presas en el } \\
\text { suelo }\end{array}$ \\
\hline Turdus grayi & $\mathrm{R}$ & $\mathrm{BJ}, \mathrm{BE}, \mathrm{SBC}$ & $\mathrm{Rr}$ & & & & $\begin{array}{l}\text { Insectívoro - recoge presas en el } \\
\text { suelo }\end{array}$ \\
\hline Turdus rufopalliatus & $\mathrm{R}$ & $\mathrm{BP}$ & $\mathrm{Rr}$ & & & $\mathrm{E}$ & $\begin{array}{l}\text { Insectívoro - recoge presas en el } \\
\text { suelo }\end{array}$ \\
\hline Turdus migratorius & MI & BPE, BE & $\mathrm{Cm}$ & & & & $\begin{array}{l}\text { Insectívoro - recoge presas en el } \\
\text { suelo }\end{array}$ \\
\hline \multicolumn{8}{|l|}{ Mimidae } \\
\hline Melanotis caerulescens & $\mathrm{R}$ & $\mathrm{BP}$ & $\mathrm{Cm}$ & & & $\mathrm{E}$ & $\begin{array}{l}\text { Insectívoro - recoge presas en el } \\
\text { suelo }\end{array}$ \\
\hline Toxostoma curvirostre & $\mathrm{R}$ & BJ, BPE, SBC & $\mathrm{Cm}$ & & & & $\begin{array}{l}\text { Insectívoro - recoge presas en el } \\
\text { suelo }\end{array}$ \\
\hline Toxostoma longirostre & $\mathrm{R}$ & BJ & & & & $\mathrm{CE}$ & $\begin{array}{l}\text { Insectívoro - recoge presas en el } \\
\text { suelo }\end{array}$ \\
\hline Mimus polyglottos & $\mathrm{R}$ & $\mathrm{BP}$ & $\mathrm{Rr}$ & & & & $\begin{array}{l}\text { Insectívoro - recoge presas en el } \\
\text { suelo }\end{array}$ \\
\hline \multicolumn{8}{|l|}{ Bombycilliidae } \\
\hline Bombycilla cedrorum & MI & BJ & & & & & $\begin{array}{l}\text { Frugívoro - recolector de dosel } \\
\text { superior }\end{array}$ \\
\hline \multicolumn{8}{|l|}{ Ptiliogonatidae } \\
\hline Ptiliogonys cinereus & $\mathrm{R}$ & BPE, BP & $\mathrm{Cm}$ & & & $\mathrm{CE}$ & $\begin{array}{l}\text { Insectívoro - captura presas en el } \\
\text { aire arriba del dosel }\end{array}$ \\
\hline Phainopepla nitens & MI & MX & & & & & $\begin{array}{l}\text { Insectívoro - captura presas en el } \\
\text { aire debajo del dosel }\end{array}$ \\
\hline \multicolumn{8}{|l|}{ Peucedramidae } \\
\hline Peucedramus taeniatus & $\mathrm{R}$ & BPE, BP & $\mathrm{Cm}$ & & & & $\begin{array}{l}\text { Insectívoro - recolector en el follaje } \\
\text { de bajo del dosel }\end{array}$ \\
\hline \multicolumn{8}{|l|}{ Passeridae } \\
\hline Passer domesticus & $\mathrm{R}$ & BPE,BP, BE & $\mathrm{Cm}$ & & & & $\begin{array}{l}\text { Granívoro - recolector en el suelo o } \\
\text { bajo el suelo }\end{array}$ \\
\hline \multicolumn{8}{|l|}{ Fringillidae } \\
\hline Euphonia elegantissima & $\mathrm{R}$ & BPE & $\mathrm{Cm}$ & & & & $\begin{array}{l}\text { Frugívoro - recolector de suelo a } \\
\text { dosel bajo }\end{array}$ \\
\hline Haemorhous mexicanus & $\mathrm{R}$ & BJ, BPE, BP & $\mathrm{Ab}$ & & & & $\begin{array}{l}\text { Granívoro - recolector en el suelo o } \\
\text { bajo el suelo }\end{array}$ \\
\hline Haemorhous cassinii & MI & BPE & $\mathrm{Rr}$ & & NT & & $\begin{array}{l}\text { Granívoro - recolector en el suelo o } \\
\text { bajo el suelo }\end{array}$ \\
\hline Loxia curvirostra & $\mathrm{R}$ & $\mathrm{BP}$ & $\mathrm{Cm}$ & & & & $\begin{array}{l}\text { Granívoro - recolector de dosel bajo } \\
\text { a superior }\end{array}$ \\
\hline Spinus spinus & $\mathrm{R}$ & BPE & & & & & $\begin{array}{l}\text { Granívoro - recolector de dosel bajo } \\
\text { a superior }\end{array}$ \\
\hline
\end{tabular}


Orden

Estacionalidad Hábitat

Abundancia NOM-059 IUCN Endemismo Gremio

Familia

Especie

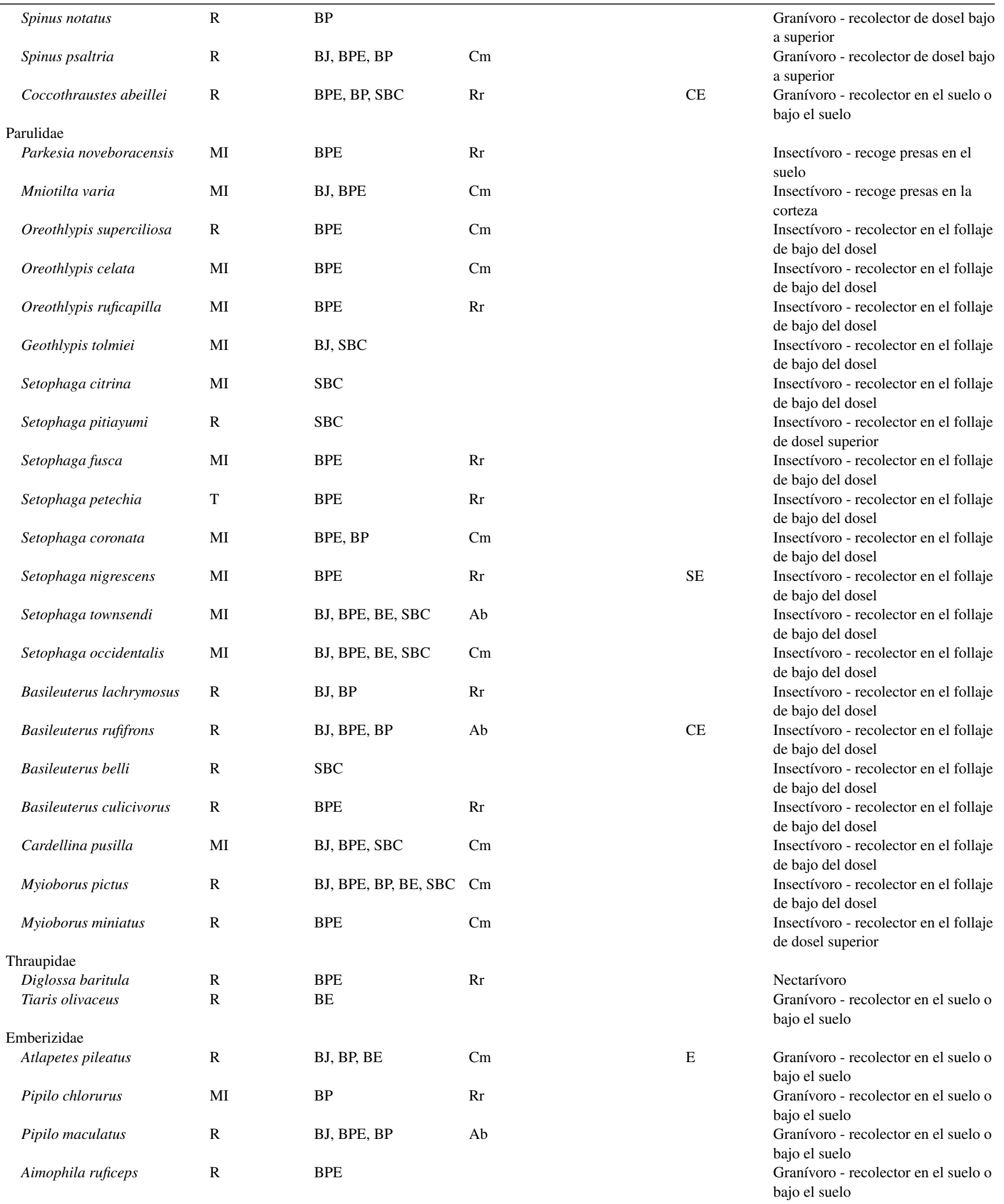




\begin{tabular}{|c|c|c|c|c|c|c|c|}
\hline $\begin{array}{l}\text { Orden } \\
\text { Familia } \\
\text { Especie }\end{array}$ & Estacionalidad & Hábitat & Abundancia & NOM-059 & IUCN & Endemismo & Gremio \\
\hline Melozone fusca & $\mathrm{R}$ & BJ, BPE, BP & $\mathrm{Ab}$ & & & & $\begin{array}{l}\text { Granívoro - recolector en el suelo o } \\
\text { bajo el suelo }\end{array}$ \\
\hline Peucaea botterii & $\mathrm{R}$ & BJ & & & & & $\begin{array}{l}\text { Granívoro - recolector en el suelo o } \\
\text { bajo el suelo }\end{array}$ \\
\hline Oriturus superciliosus & $\mathrm{R}$ & BPE & $\mathrm{Rr}$ & & & $\mathrm{E}$ & $\begin{array}{l}\text { Granívoro - recolector en el suelo o } \\
\text { bajo el suelo }\end{array}$ \\
\hline Spizella passerina & MV & BJ & $\mathrm{Cm}$ & & & & $\begin{array}{l}\text { Granívoro - recolector en el suelo o } \\
\text { bajo el suelo }\end{array}$ \\
\hline Melospiza melodia & $\mathrm{R}$ & BPE & & & & & $\begin{array}{l}\text { Granívoro - recolector en el suelo o } \\
\text { bajo el suelo }\end{array}$ \\
\hline Junco phaeonotus & $\mathrm{R}$ & $\mathrm{BJ}, \mathrm{BPE}, \mathrm{BP}$ & $\mathrm{Ab}$ & & & $\mathrm{CE}$ & $\begin{array}{l}\text { Granívoro - recolector en el suelo o } \\
\text { bajo el suelo }\end{array}$ \\
\hline \multicolumn{8}{|l|}{ Cardinalidae } \\
\hline Piranga flava & $\mathrm{R}$ & BJ, BPE, BP, BE, SBC & $\mathrm{Ab}$ & & & & $\begin{array}{l}\text { Insectívoro - recolector en el follaje } \\
\text { de dosel superior }\end{array}$ \\
\hline Piranga rubra & MI & $\mathrm{BJ}, \mathrm{BP}$ & $\mathrm{Rr}$ & & & & $\begin{array}{l}\text { Insectívoro - recolector en el follaje } \\
\text { de dosel superior }\end{array}$ \\
\hline Piranga ludoviciana & MI & BP & $\mathrm{Rr}$ & & & & $\begin{array}{l}\text { Insectívoro - recolector en el follaje } \\
\text { de dosel superior }\end{array}$ \\
\hline Piranga bidentata & $\mathrm{R}$ & BPE, BP, SBC & $\mathrm{Rr}$ & & & & $\begin{array}{l}\text { Insectívoro - recolector en el follaje } \\
\text { de dosel superior }\end{array}$ \\
\hline Rhodothraupis celaeno & $\mathrm{R}$ & $\mathrm{BP}$ & $\operatorname{Rr}$ & & & $\mathrm{E}$ & $\begin{array}{l}\text { Granívoro - recolector de dosel bajo } \\
\text { a superior }\end{array}$ \\
\hline Cardinalis cardinalis & $\mathrm{R}$ & SBC & & & & & $\begin{array}{l}\text { Insectívoro - recolector en el follaje } \\
\text { de dosel superior }\end{array}$ \\
\hline Pheucticus ludovicianus & MI & $\mathrm{BE}$ & & & & & $\begin{array}{l}\text { Insectívoro - recolector en el follaje } \\
\text { de dosel superior }\end{array}$ \\
\hline Pheucticus melanocephalus & $\mathrm{R}$ & $\mathrm{BJ}, \mathrm{BE}, \mathrm{SBC}$ & $\mathrm{Cm}$ & & & SE & $\begin{array}{l}\text { Insectívoro - recolector en el follaje } \\
\text { de dosel superior }\end{array}$ \\
\hline Passerina caerulea & $\mathrm{R}$ & SBC & & & & & $\begin{array}{l}\text { Granívoro - recolector de dosel bajo } \\
\text { a superior }\end{array}$ \\
\hline Passerina versicolor & $\mathrm{R}$ & SBC & & & & SE & $\begin{array}{l}\text { Granívoro - recolector de dosel bajo } \\
\text { a superior }\end{array}$ \\
\hline \multicolumn{8}{|l|}{ Icteridae } \\
\hline Quiscalus mexicanus & $\mathrm{R}$ & BPE & $\mathrm{Cm}$ & & & & Omnívoro - terrestre \\
\hline Molothrus ater & $\mathrm{R}$ & BP & $\mathrm{Cm}$ & & & & $\begin{array}{l}\text { Granívoro - recolector de dosel bajo } \\
\text { a superior }\end{array}$ \\
\hline Icterus wagleri & $\mathrm{R}$ & SBC & & & & & $\begin{array}{l}\text { Insectívoro - recolector en el follaje } \\
\text { de bajo del dosel }\end{array}$ \\
\hline Icterus cucullatus & $\mathrm{T}$ & BPE & $\mathrm{Rr}$ & & & SE & $\begin{array}{l}\text { Insectívoro - recolector en el follaje } \\
\text { de dosel superior }\end{array}$ \\
\hline Icterus gularis & $\mathrm{R}$ & BPE & & & & & $\begin{array}{l}\text { Insectívoro - recolector en el follaje } \\
\text { de dosel superior }\end{array}$ \\
\hline Icterus graduacauda & $\mathrm{R}$ & BJ, BPE, SBC & $\mathrm{Cm}$ & & & $\mathrm{CE}$ & $\begin{array}{l}\text { Insectívoro - recolector en el follaje } \\
\text { de dosel superior }\end{array}$ \\
\hline Icterus galbula & MI & BJ & $\operatorname{Rr}$ & & & & $\begin{array}{l}\text { Insectívoro - recolector en el follaje } \\
\text { de dosel superior }\end{array}$ \\
\hline Icterus parisorum & $\mathrm{R}$ & $\mathrm{BJ}, \mathrm{BPE}$ & $\mathrm{Cm}$ & & & $\mathrm{SE}$ & $\begin{array}{l}\text { Insectívoro - recolector en el follaje } \\
\text { de dosel superior }\end{array}$ \\
\hline
\end{tabular}

\section{Referencias}

Amadon, D. y Phillips, A. R. (1947). Notes on Mexican birds. The Auk, 64 576-581.

AOU (American Ornithologistsí Union). (1998). Checklist of North American birds (7th ed.). Washington, D.C: American Ornithologists' Union.

Arizmendi, M. C., y Márquez-Valdelamar, L. (Eds.). (1999). Áreas de importancia para la conservación de las aves en México. México, D.F.: CIPAMEX.

Berlanga, H., Gómez-de Silva, H., Vargas-Canales, V. M., RodríguezContreras, V., Sánchez-González, L. A., Ortega-Álvarez, R., et al. (2015). Aves de México: lista actualizada de especies y nombres comunes.
México D.F.: Comisión Nacional para el Conocimiento y Uso de la Biodiversidad.

Bezaury-Creel, J., Gutiérrez, D., Remolina, J. F., Pérez, J. J., González, J., Betancourt, N., et al. (2009). Áreas naturales protegidas y desarrollo social en México. In Capital natural de México. Vol II: Estado de conservación y tendencias de cambio. pp. 385-431. México, D.F.: Comisión Nacional para el Conocimiento y Uso de la Biodiversidad.

BirdLife. (2016). Important bird and biodiversity areas (IBAs). Recuperado 12 Jul 2016 de http://www.birdlife.org/datazone/info/ibacriteria

Buckland, S. T. (2006). Point-transect surveys for songbirds: robust methodologies. The Auk, 123, 45-357. 
Carabias, J., Provencio, E., de la Maza, J. y Ruiz, M. I. (1999). Programa de manejo Reserva de la Biosfera Sierra Gorda. México, D.F.: Instituto Nacional de Ecología.

Carbó-Ramírez, P. y Zuria, I. (2011). The value of small urban greenspaces for birds in a Mexican city. Landscape and Urban Planning, 100, 213-222.

Ceballos, G., y Márquez, L. (Eds.). (2000). Las aves de México en peligro de extinción. México, D.F.: Universidad Nacional Autónoma de México, Comisión Nacional para el Conocimiento y Uso de la Biodiversidad, y Fondo de Cultura Económica.

Colwell, R.K. (2013). EstimateS: statistical estimation of species richness and shared species from samples. Ver. 9.1 User's guide and application. Disponible en: http://viceroy.eeb.uconn.edu/EstimateS/index.html

Colwell, R. K. y Coddington, J. A. (1994). Estimating terrestrial biodiversity through extrapolation. Philosophical Transaction Royal Society Series B, $345,101-118$.

Conabio (Comisión Nacional para el Conocimiento y Uso de la Biodiversidad). (2002). Criterios utilizados en la designación de las AICAS. Recuperado 24 Jun 2016 de http://conabioweb.conabio. gob.mx/aicas/doctos/criterios-aicas.html

Conanp (Comisión Nacional de Áreas Naturales Protegidas). (2007). Estudio previo justificativo para la modificación del decreto por el que se pretende re-categorizar el Parque Nacional Los Mármoles como Área de Protección de Flora y Fauna. México, D.F.: Secretaria del Medio Ambiente y Recursos Naturales.

Conanp (Comisión Nacional de Áreas Naturales Protegidas). (2013). Monitoreo de avifauna en la laguna de Metztitlán y bosque de sabino (Juniperus flaccida) en la Reserva de la Biosfera Barranca de Metztitlán, Hidalgo. México, D.F.: Secretaría del Medio Ambiente y Recursos Naturales.

Conanp (Comisión Nacional de Áreas Naturales Protegidas). (2015). Áreas Naturales Protegidas Decretadas. Recuperado 24 Jun 2016 de http://www.gob.mx/conanp/acciones-y-programas/areas-naturalesprotegidas-decretadas?idiom $=\mathrm{es}$

Cruz-Elizalde, R., Ramírez-Bautista, A., Wilson, L. D. y Hernández-Salinas, U. (2015). Effectiveness of protected areas in herpetofaunal conservation in Hidalgo, Mexico. Herpetological Journal, 25, 41-48.

Del Hoyo, J., Elliot, A., y Sargatal, J. (Eds.). (1992). Handbook of the birds of the World, Ostrich to Ducks (1). Barcelona: Birdlife International y Lynx.

Del Hoyo, J., Elliot, A., y Sargatal, J. (Eds.). (1996). Handbook of the birds of the world, Hoatzin to Auks (3). Barcelona: Birdlife International y Lynx.

Del Hoyo, J., Elliot, A., y Sargatal, J. (Eds.). (1999). Handbook of the birds of the world, Barn-owls to Hummingbirds (5). Barcelona: Birdlife International y Lynx.

Del Hoyo, J., Elliot, A., y Sargatal, J. (Eds.). (2005). Handbook of the birds of the world, Cuckoo-shrikes to Thrushes (10). Barcelona: Birdlife International y Lynx.

Emlen, J. T. (1971). Population densities of birds derived from transect counts. The Auk, 88, 323-342.

Espinoza, D., Aguilar, C. y Ocegueda, S. (2004). Identidad biogeográfica de la sierra Madre Oriental y posibles subdivisiones bióticas. En I. Luna, J. J. Morrone, y D. Espinosa (Eds.), Biodiversidad de la sierra Madre Oriental (pp. 487-500). México, D.F: Comisión Nacional para el Conocimiento y Uso de la Biodiversidad, Las Prensas de Ciencias, Universidad Nacional Autónoma de México.

Friedmann, H., Griscom, L. y Moore, R. T. (1950). Distributional check-list of the birds of Mexico. Part I. Pacific Coast Avifauna, 29, 1-202.

Gallina, M. P., González, A., Mortal, R. C. y Tello, G. C. (1974). Bases para la reestructuración del Parque Nacional "El Chico", Hidalgo México (Tesis). México, D.F.: Facultad de Ciencias, Universidad Nacional Autónoma de México.

García, I. y Ortiz-Pulido, R. (2007). Determinación de aves indicadoras de la calidad ambiental en bosque de encino (Quercus sp., Fagaceae) en las cercanías del Parque Nacional El Chico Hidalgo, México. En G. Pulido-Flores y A. L. López-Escamilla (Eds.), IV Foro de investigadores para la conservación y II Simposio de áreas naturales protegidas en el estado de Hidalgo (p. 17). Pachuca, Hidalgo: Universidad Autónoma del Estado de Hidalgo.

García-Sánchez, C. A., Sánchez-González, A. y Villaseñor, J. L. (2014). La familia Asteraceae en el Parque Nacional Los Mármoles, Hidalgo, México. Acta Botanica Mexicana, 106, 97-116.
Gómez-de Silva, H. y Medellín, R. (2001). Evaluating completeness of species lists for conservation and macroecology: a case study of mexican land birds. Conservation Biology, 15, 1384-1395.

González-García, F. y Gómez-de Silva, H. (2003). Especies endémicas: riqueza patrones de distribución y retos para su conservación. En H. Gómez-de Silva y A. Oliveras-de Ita (Eds.), Conservación de aves. Experiencias en México (pp. 150-194). México, D.F.: CIPAMEX, Comisión Nacional para el Conocimiento y Uso de la Biodiversidad, National Fish and Wildlife Foundation.

González-Salazar, C., Martínez-Meyer, E. y López-Santiago, G. (2014). A hierarchical classification of trophic guilds for North American birds and mammals. Revista Mexicana de Biodiversidad, 85, 931-941.

Guzmán, E. (2015). Sub-red de interacciones colibrí-planta en el Parque Nacional Los Mármoles, Hidalgo México (tesis). Pachuca, Hidalgo, México: Instituto de Ciencias Básicas e Ingeniería, Universidad Autónoma del Estado de Hidalgo.

Hernández-Flores, S. D. y Rojas-Martínez, A. E. (2010). Lista actualizada y estado de conservación de los mamíferos del Parque Nacional El Chico, Hidalgo, México. Acta Zoológica Mexicana (nueva serie), 26, 563-583.

Herzog, S. K., Kessler, M. y Cahill, T. M. (2002). Estimating species richness of tropical bird communities from rapid assessment data. The Auk, 119, 749-769.

Howell, S. N. G. y Webb, S. (1995). A guide to the birds of Mexico and Northern Central America. New York: Oxford University Press.

Inegi (Instituto Nacional de Estadística y Geografía). (2016). Recursos Naturales: uso de suelo y vegetación. Datos vectoriales escala 1:250 000 serie V. Recuperado 7 Jun 2016, de: http://www.inegi.org.mx/geo/contenidos/recnat/usosuelo/

IUCN (International Union for Conservation of Nature). (2016). The IUCN red list of threatened species. Recuperado 24 Jun 2016 de http://www.iucnredlist.org/

Leopold, A. S. (1965). Fauna silvestre de México. México, D.F.: Instituto Mexicano de Recursos Naturales Renovables (IMERNAR).

Magurran, A. E. (2004). Measuring biological diversity. Oxford: Blackwell Publishing.

Mancilla, M. (1988). Estudio preliminar de la avifauna en el transecto Zacualtipán-Zoquizoquipan-San Juan Metztitlán en el este de Hidalgo México (Tesis). México, D.F.: ENEP Iztacala, Universidad Nacional Autónoma de México.

Margules, C. R. y Sarkar, S. (2009). Planeación sistemática de la conservación. México, D.F.: Universidad Nacional Autónoma de México, Comisión Nacional de Áreas Naturales Protegidas, y Comisión Nacional para el Conocimiento y Uso de la Biodiversidad.

Martínez-García, V. (2006). Interacciones colibrí-planta en tres tipos de vegetación de la Reserva de la Biosfera Barranca de Metztitlán, Hidalgo México (Tesis). Pachuca, Hidalgo, México: Universidad Autónoma del Estado de Hidalgo.

Martínez-Morales, M. A. (2001). Forest fragmentation effects on bird communities of tropical montane cloud forests in eastern México (Tesis doctoral). Cambridge, Reino Unido: University of Cambridge.

Martínez-Morales, M. A. (2004). Nuevos registros de aves en el bosque mesófilo de montaña del noreste de Hidalgo, México. Huitzil, 5, 12-19.

Martínez-Morales, M. A., Mendiola, V., Zuria, I., Chávez, M. C. y Campuzano, R. G. (2013). La conservación de las aves más allá de las áreas naturales protegidas: el caso de la avifauna del Rancho Santa Elena Hidalgo. Huitzil, $14,87-100$.

Martínez-Morales, M. A., Ortiz-Pulido, R., de la Barrera, B., Zuria, I. L., BravoCadena, J. y Valencia-Herverth, J. (2007). Hidalgo. En R. Ortiz-Pulido, A. Navarro-Sigüenza, H. Gómez-de Silva, O. Rojas-Soto, y T. A. Peterson (Eds.), Avifaunas estatales de México (pp. 49-51). Pachuca: CIPAMEX.

Mengel, R. y Warner, D. (1948). Golden Eagles in Hidalgo, Mexico. The Wilson Bulletin, 60, 122 .

Moreno, C. E. (2001). Métodos para medir la biodiversidad. Zaragoza: M y T - Manuales y Tesis SEA.

National Geographic Society. (2011). Field guide to the birds of North America. Washington D.C: National Geographic Society.

Navarro, A. G. y Peterson, A. T. (2007). Mapas de las aves de México basados en WWW. México, D.F: Informe final SNIB-Conabio proyecto Núm. CE015. 
Navarro-Sigüenza, A. G., Rebón-Gallardo, M. F., Gordillo-Martínez, A., Peterson, A. T., Berlanga, H. y Sánchez-González, L. A. (2014). Biodiversidad de aves en México. Revista Mexicana de Biodiversidad, 85(Supl.), S476-S495.

Ortiz-Pulido, R., Bravo-Cadena, J., Martínez-García, V., Reyes, D., MendiolaGonzález, M., Sánchez, G., et al. (2010). Avifauna de la Reserva de la Biosfera Barranca de Metztitlán, Hidalgo, México. Revista Mexicana de Biodiversidad, 81, 373-391.

Ortiz-Pulido, R. y Díaz, R. (2001). Distribución de colibríes en la zona baja del centro de Veracruz México. Ornitología Neotropical, 12, 297-317.

Ortiz-Pulido, R., Gómez-de Silva, H., González-García, F. y Álvarez, A. (1995). Avifauna del Centro de Investigaciones Costeras La Mancha, Veracruz México. Acta Zoológica Mexicana (nueva serie), 66, 87-118.

Ortiz-Pulido, R., Mauricio-López, E., Martínez-García, V. y Bravo-Cadena, J. (2008). ¿Sabes quién vive en el Parque Nacional El Chico? Colibríes. Pachuca, Hidalgo, México: Universidad Autónoma del Estado de Hidalgo, Secretaría de Educación Pública Hidalgo, Dirección del Parque Nacional El Chico.

Pavón, N. P. y Meza, M. (2009). Cambio climático en el estado de Hidalgo: clasificación y tendencias climáticas. Pachuca, Hidalgo, México: Universidad Autónoma del Estado de Hidalgo.

Peterson, R. T. y Chalif, E. L. (2008). Aves de México guía de campo. México D.F.: Editorial Diana.

Pineda-López, R., Arellano-Sanaphre, A. y la Vega-Aguirre, D. (2013). Registros nuevos y notables de aves acuáticas en Querétaro y estados adyacentes. Huitzil, 14, 1-6.

Ralph, C. J., Geupel, G., Pyle, P., Martin, T., DeSante, D. y Milá, B. (1996) Manual de métodos de campo para el monitoreo de aves terrestres. Albany, California: General Technical Report PSW-GTR-159. Pacific Southwest Research Station, Forest Service, U.S. Departament of Agriculture.

Ramírez-Albores, J. E. (2006). Variación en la composición de comunidades de aves en la Reserva de la Biosfera Montes Azules y áreas adyacentes, Chiapas México. Biota Neotropica, 6, 1-19.

Ramírez-Cruz, S., Sánchez-González, A. y Tejero-Diez, D. (2009). La pteridoflora del Parque Nacional Los Mármoles, Hidalgo México. Boletín de la Sociedad Botánica Mexicana, 84, 35-44.

Randell, J. (2008). Ordenamiento ecológico territorial regional en los municipios donde se ubica el Parque Nacional Los Mármoles. México, D.F. Consejo Estatal de Ecología. Informe final SNIB-Conabio proyecto Núm. DQ006.

Rodríguez-Yáñez, C. A., Villalón, R. y Navarro, A. G. (1994). Bibliografía de las aves de México (1825-1992). Publicaciones especiales del Museo de Zoología Núm. 8. México, D.F.: Facultad de Ciencias, Universidad Nacional Autónoma de México.

Rojas-Soto, O. R., Sánchez-González, L. A. y López de Aquino, S. (2002). New information on the birds of northern Hidalgo, Mexico. The Southwestern Naturalist, 47, 471-475.
Rzedowski, J. (1978). Vegetación de México. México, D.F.: Limusa.

Sánchez-González, L. A. (2013). Cuando un" nuevo registro" es realmente un nuevo registro: consideraciones para su publicación. Huitzil, 14, $17-21$.

Sánchez-González, A., Álvarez, E. y Tejero-Diez, J. D. (2010). Richness and distribution patterns of Ferns and Lycopods in Los Mármoles National Park, Hidalgo, Mexico. Journal of the Torrey Botanical Society, 137, 373-379.

Sánchez-Mejorada, H. (1978). Manual de campo de las cactáceas y suculentas de la barranca de Metztitlán. Publicación de Difusión Cultural Núm. 2. México D.F.: Sociedad Mexicana de Cactología-Conacyt.

Semarnat (Secretaría del Medio Ambiente y Recursos Naturales). (2010). Norma Oficial Mexicana NOM-059-SEMARNAT-2010, Protección ambiental Especies nativas de México de flora y fauna silvestres - Categorías de riesgo y especificaciones para su inclusión, exclusión o cambio - Lista de especies en riesgo. Diario Oficial de la Federación. 30 de diciembre de 2010, Segunda Sección, México.

Sutton, G. M. y Burleigh, T. D. (1940). A new warbling vireo from Hidalgo. The Auk, 57, 398-400.

Sutton, G. M. y Burleigh, T. D. (1941). Birds recorded in the state of Hidalgo, Mexico, by the sample expedition of 1939. Annals of the Carnaige Museum of Pittsburgh, 28, 169-186.

Valencia-Herverth, J., Ortiz-Pulido, R. y Enríquez, P. (2012). Riqueza y distribución espacial de rapaces nocturnas en Hidalgo, México. Huitzil, 13, $116-129$.

Valencia-Herverth, R. y Valencia-Herverth, J. (2008). Inventario preliminar de la ornitofauna del municipio de Jacala, Hidalgo. TecnoINTELECTO, 5, 20-24.

Valencia-Herverth, J., Valencia-Herverth, R., Mendiola-González, M. E., Sánchez-Cabrera, M. y Martínez-Morales, M. A. (2011). Registros nuevos y sobresalientes para el estado de Hidalgo, México. Acta Zoológica Mexicana (nиeva serie), 27, 843-861.

Valencia-Herverth, R., Valencia-Herverth, J. y Mendoza-Quijano, F. (2010). Registros nuevos para la avifauna del estado de Hidalgo, México. Revista Mexicana de Biodiversidad, 81, 205-208.

Valencia-Herverth, R., Valencia-Herverth, J., Mendoza-Quijano, F., Linarte, A. y Hernández, L. (2008). Avifauna de dos fragmentos de selva mediana subperennifolia en la región Huasteca del estado de Hidalgo, México. TecnOINTELECTO, 5, 12-19.

Valencia-Herverth, R., Valencia-Herverth, J., Padilla, R., Olivares, M., Hernández, R. y Olivares, M. (2012). Información adicional sobre la avifauna de Hidalgo, México. Huitzil, 13, 95-103.

Villada, M. M. (1865). Estudios sobre la fauna de Pachuca, Real del Monte, Mineral del Chico y Barranca Honda. En R. Almaraz (Ed.), Memoria de los trabajos ejecutados por la Comisión Científica de Pachuca en el año 1864 Edición facsimilar 1993. (pp. 261-345). Pachuca, Hidalgo, México: Universidad Autónoma del Estado de Hidalgo. 